\title{
STRING THEORY IN THE NAMBU-GOTO FORMALISM AND NONCOMMUTATIVITY
}

\author{
N. Mansour ${ }^{1}$, E. Diaf ${ }^{2}$, M. B. Sedra ${ }^{1}$ \\ ${ }^{1}$ SIMO-Lab, Faculty of Sciences, Ibn Tofail University, \\ Kenitra, Morocco \\ ${ }^{2}$ Department of Physics, OLMAN-RL Pluridisciplinary Faculty of Nador, \\ University Mohammed First, Oujda B.P N 300, \\ Selouane, 27000 Nador, Morocco \\ e-mail:nmansour@live.fr,eldiaf@gmail.commysedra@yahoo.fr \\ (Received September 13, 2018; in final form - January 19, 2019)
}

\begin{abstract}
In this paper, we studied the string theory in its original form of Nambu-Goto. We found that the calculation of the variation of the Nambu-Goto action with respect to the metric of the word sheet leads to a nonlinear action which is not strictly equivalent to the Polyakov action. Then the equation of motion corresponding to this action is nonlinear. This was the reason why we decided to solve the nonlinear Nambu-Goto equation of motion directly. This has been done for a string interacting with a constant, antisymmetric tensor field B in the Nambu-Goto formulation, and we derive the noncommutativity among the string end points coordinates.
\end{abstract}

Key words: string theory, Nambu-Goto formalism, noncommutative geometry.

DOI: https://doi.org/10.30970/jps.23.1103

PACS number(s): 11.25.Yb; 11.25.-w; 11.25.Hf; 02.40.Gh; 11.25.Uv

\section{INTRODUCTION}

In mathematical physics, noncommutative geometry [1] studies the geometry of "quantum space" [2], in which the coordinate functions are noncommutative, satisfying a noncommutative algebra of the form:

$$
\left[X^{\mu}, X^{\nu}\right]=i \Theta^{\mu \nu}
$$

where $\Theta^{\mu \nu}$ is an antisymmetric constant tensor.

String theory $[3,4,5,6]$, is a quantum theory of one-dimensional objects called strings which are of two different types: open and closed. Geometrically, open strings are characterized by free endpoints, whereas closed strings are characterized by connected endpoints. The study of noncommutativity has a long history in physics, and it attracted much attention in the past few years owing to the inspiration from the string theory (for a review and more references see $[7,8,9,10,11$, $12,13,14])$. In the presence of an antisymmetric tensor background field $B_{\mu \nu}$, an open string moving with mixed Neumann and Dirichlet boundary conditions will make the spacetime coordinates noncommutative, which is manifested in the noncommutativity at the end points of the string which are attached to $D$-branes and obey canonical commutation relations (1). Different approaches have been adopted to obtain this result $[15,16,17,18$, $19,20,26]$. We establish explicitly that the Polyakov action is not strictly equivalent to the Nambu-Goto action, so we have to solve the nonlinear equation of motion of Nambu-Goto action directly, and derive the noncommutativity from the string end points coordinates.

\section{NAMBU-GOTO AND POLYAKOV STRING ACTIONS. ARE THEY STRICTLY EQUIVALENT?}

A string is a 1-dimensional object moving in a $D$ dimensional space-time (1 time, $D-1$ space dimensions). As the string moves in time, it sweeps out a 2dimensional area in a $D$-dimensional space-time; this is called the worldsheet of the string denoted $\Sigma$. This string is parametrized by a parameter $\sigma,(0<\sigma<l$ for some finite $l)$. As the string moves in time, every point on the string describes a trajectory in space-time, this trajectory can be parameterized by a variable $\tau$. $\tau$ could have the range $-\infty<\tau<+\infty$. Every point on the worldsheet is parameterized by the pair $(\sigma, \tau)$. In the Lagrangian formalism, one can consider maps

$$
x: \Sigma \rightarrow X^{\mu} .
$$

The space-time position of a point $(\tau, \sigma)$ on the string worldsheet $\Sigma$ is given by the function $X^{\mu}(\tau, \sigma),(\mu=$ $0,1, \ldots, D-1)$.

Let us consider that the action for the Nambu-Goto string $(\hbar=c=1)$ in a space-time with the flat Minkowskian metric $\eta_{\mu \nu}$ is proportional to the area of the world-sheet swept out by the string during its motion:

$$
S_{\mathrm{NG}}=-T \int_{\sigma 1}^{\sigma 2} d \tau \int_{0}^{\pi} d \sigma \sqrt{-h(\tau, \sigma)}, \quad L=-T \sqrt{-h}
$$


where $T=\frac{1}{2 \pi \alpha^{\prime}}$ is called the string tension and

$$
\begin{aligned}
& -h=-\operatorname{det}\left(h_{a b}\right)=\left(\dot{X} X^{\prime}\right)^{2}-\dot{X}^{2} X^{\prime 2} \geq 0 \\
& h_{a b}=\left(\partial_{a} X^{\mu} \partial_{b} X_{\mu}\right)=\left(\begin{array}{cc}
\dot{X}^{2} & \dot{X} X^{\prime} \\
\dot{X} X^{\prime} & \left(X^{\prime}\right)^{2}
\end{array}\right), a, b=0,1 \\
& h^{a b}=\frac{1}{h}\left(\begin{array}{cc}
X^{\prime 2} & -\dot{X} X^{\prime} \\
-\dot{X} X^{\prime} & \dot{X}^{2}
\end{array}\right)
\end{aligned}
$$

Here, the "dot" and "prime" denote derivatives with respect to time $\tau$ and the space coordinate $\sigma$, respectively, as follows:

$$
\begin{aligned}
& \dot{X}^{\mu} \equiv \partial_{0} X^{\mu} \equiv \partial X^{\mu} / \partial \tau \\
& X^{\prime \mu} \equiv \partial_{1} X^{\mu} \equiv \partial X^{\mu} / \partial \sigma
\end{aligned}
$$

The condition $-h \geq 0$ means that the surface swept out by the string in the space-time is everywhere time-like or null. The strip $0 \leq \sigma \leq \pi$ is mapped on the world-sheet spanned by the string in the Minkowski space, which is described by the coordinates $X(\tau, \sigma)$ in an arbitrary inertial frame.
The Polyakov action is:

$$
S_{\sigma}=\frac{T}{2} \int d^{2} \sigma \sqrt{-h} h^{a b} g_{\mu \nu} \partial_{a} X^{\mu} \partial_{b} X^{\mu} .
$$

In the Minkowski space $h^{a b}=\eta^{a b}=\operatorname{diag}(1,-1)$ we have:

$$
\begin{aligned}
S_{\sigma} & =\frac{T}{2} \int d^{2} \sigma \sqrt{-h} h^{a b} g_{\mu \nu} \partial_{a} X^{\mu} \partial_{b} X^{\mu} \\
& =\frac{T}{2} \int_{\Sigma}\left(\dot{X}^{2}-X^{\prime 2}\right) d^{2} \sigma .
\end{aligned}
$$

Varying the action (9) with respect to the metric $h^{a b}$ yields the stress energy tensor $T_{a b}$

$$
\delta S_{\sigma}=\frac{T}{2} \int d^{2} \sigma\left(\delta(\sqrt{-h}) h^{a b}+\sqrt{-h} \delta h^{a b}\right) g_{\mu \nu} \partial_{a} X^{\mu} \partial_{b} X^{\mu} .
$$

To calculate the variation of this action, where there is a square root in its form, we first prove that $\delta(\sqrt{-h})=$ $\frac{1}{2} \sqrt{-h} h_{a b} \delta h^{a b}$ as follows:

$$
\begin{aligned}
\operatorname{det}\left(h_{a b}\right) & =e^{\operatorname{trln}\left(h_{a b}\right)} \\
\operatorname{det}\left(h_{a b}+\delta h_{a b}\right) & =e^{\operatorname{trln}\left(h_{a b}+\delta h_{a b}\right)}=e^{\operatorname{trln}\left[h_{a b}\left(1+h_{a b}^{-1} \delta h_{a b}\right)\right]} \\
& =e^{\operatorname{trln}\left(h_{a b}\right)+\operatorname{trln}\left(1+h_{a b}^{-1} \delta h_{a b}\right)}=\operatorname{det}\left(h_{a b}\right) e^{\operatorname{trln}\left(1+h_{a b}^{-1}+\delta h_{a b}\right)} \\
& =\operatorname{det}\left(h_{a b}\right) e^{\operatorname{tr}\left(h_{a b}^{-1} \delta h_{a b}\right)} \\
& =\operatorname{det}\left(h_{a b}\right)\left(1+\operatorname{tr}\left(h_{a b}^{-1} \delta h_{a b}+\ldots\right)\right) \\
\operatorname{det}\left(h_{a b}+\delta h_{a b}\right) & =\operatorname{det}\left(h_{a b}\right)+\operatorname{det}\left(h_{a b}\right) h^{a b}\left(\delta h_{a b}\right) \operatorname{det}\left(h_{a b}+\delta h_{a b}\right)-\operatorname{det}\left(h_{a b}\right) \\
& =-\operatorname{det}\left(h_{a b}\right) \delta h_{a b} h^{a b}, \\
& \frac{\partial \operatorname{det}\left(h_{a b}\right)}{\partial h_{a b}}=-\operatorname{det}\left(h_{a b}\right) h_{a b}, \\
& \frac{\partial \sqrt{-\operatorname{det}\left(h_{a b}\right)}}{\partial h^{a b}}=\frac{1}{2} \frac{\partial\left(-\operatorname{det}\left(h_{a b}\right)\right)}{\partial h^{a b}} \frac{1}{\sqrt{-\operatorname{det}\left(h_{a b}\right)}} \\
& =-\frac{1}{2} \operatorname{det}\left(h_{a b}\right) \frac{1}{\sqrt{-\operatorname{det}\left(h_{a b}\right)}} .
\end{aligned}
$$

Finally,

$$
\frac{\partial \sqrt{-h}}{\partial h_{a b}}=\frac{-1}{2} \sqrt{-h} h_{a b}
$$

Now we can vary the Polyakov action with respect to the metric $h^{a b}$,

$$
\begin{aligned}
\frac{\delta S}{\delta h^{a b}} & =\frac{-T}{2}\left(\frac{\partial \sqrt{-h}}{\partial h^{a b}} h^{\rho \sigma}+\sqrt{-h} \frac{\partial h^{\rho \sigma}}{\partial h^{a b}}\right) g_{\mu \nu} \partial_{a} X^{\mu} \partial_{b} X^{\nu}, \\
& =\frac{-T}{2}\left(\frac{-1}{2} \sqrt{-h} h_{a b} h^{\rho \sigma}+\sqrt{-h} \frac{\partial h^{\rho \sigma}}{\partial h^{a b}}\right) g_{\mu \nu} \partial_{a} X^{\mu} \partial_{b} X^{\nu} \\
& =\frac{-T}{2} \sqrt{-h}\left(\partial_{a} X^{\mu} \partial_{b} X_{\mu}-\frac{1}{2} h_{a b}\left(h^{\rho \sigma} \partial_{\rho} X^{\mu} \partial_{\sigma} X_{\mu}\right)\right) .
\end{aligned}
$$


Then we have the stress energy tensor $T_{a b}$ :

$$
\begin{aligned}
& T_{a b}=\partial_{a} X^{\mu} \partial_{b} X_{\mu}-\frac{1}{2} h_{a b}\left(h^{\rho \sigma} \partial_{\rho} X^{\mu} \partial_{\sigma} X_{\mu}\right) \\
& T_{a b}=G_{a b}-\frac{1}{2} h_{a b}\left(h^{\rho \sigma} G_{\rho \sigma}\right),
\end{aligned}
$$

where $G_{a b}=\partial_{a} X^{\mu} \partial_{b} X_{\mu}$.

Now, the equations of motion for field $h^{a b}$ follow from setting the variation of action $S_{\sigma}$ with respect to $h^{a b}$ equal to zero, $\delta S_{\sigma}=0$, which gives:

$$
\begin{aligned}
& T_{a b}=G_{a b}-\frac{1}{2} h_{a b}\left(h^{\rho \sigma} G_{\rho \sigma}\right)=0, \\
& G_{a b}=\frac{1}{2} h_{a b}\left(h^{\rho \sigma} G_{\rho \sigma}\right), \\
& G=\frac{1}{4} h\left(h^{\alpha \beta} G_{\alpha \beta}\right)^{2},
\end{aligned}
$$

where $G=\operatorname{det}\left(G_{a b}\right), h=\operatorname{det}\left(h_{a b}\right)$ and $G_{\alpha \beta}=$ $\partial_{\alpha} X_{\mu} \partial_{\beta} X^{\mu}$. We than conclude:

$$
\sqrt{-G}=\sqrt{-h} h^{a b} h_{a b}
$$

and

$$
\int_{\Sigma} \sqrt{-G} d^{2} \sigma=\frac{1}{2} \int_{\Sigma} \sqrt{-h} h^{a b} G_{a b} d^{2} \sigma
$$

In the Minkowski space

$$
T \int_{\Sigma} \sqrt{\left(\dot{X} X^{\prime}\right)^{2}-\dot{X}^{2}\left(X^{\prime}\right)^{2}} d^{2} \sigma=\frac{T}{2} \int_{\Sigma}\left(\dot{X}^{2}-X^{\prime 2}\right) d^{2} \sigma .
$$

The right-hand side of (19) is the Polyakov action. But this is not strictly true because $\sqrt{G} d^{2} \sigma$ is a surface element of the worldsheet. Indeed we have:

$$
\sqrt{-G}=\frac{1}{2} \sqrt{-h}\left|h^{a b} G_{a b}\right| .
$$

And then:

$$
\int_{\sigma} \sqrt{-G} d^{2} \sigma=\frac{1}{2} \int_{\sigma} \sqrt{-h}\left|h^{a b} G_{a b}\right| d^{2} \sigma .
$$

In the Minkowskian space

$$
T \int_{\sigma} \sqrt{\left(\dot{X} X^{\prime}\right)^{2}-\dot{X}^{2}\left(X^{\prime}\right)^{2}} d^{2} \sigma=\frac{1}{2} \int_{\sigma}\left|\dot{X}^{2}-\left(X^{\prime}\right)^{2}\right| d^{2} \sigma .
$$

Then the equation of motion corresponding to (22) is nonlinear. This was the reason why we decided to solve the nonlinear Nambu-Goto equation directly.

\section{INTERACTING STRING IN THE NAMBU-GOTO FORMALISM: SOLUTIONS OF EQUATIONS OF MOTION OF THE NAMBU STRING}

The string sweeps out a worldsheet which may be parametrized by two parameters $\tau$ and $\sigma$. The NambuGoto action for a bosonic string moving in the presence of a constant background Neveu-Schwarz two-form field $B_{\mu \nu}$ reads [21, 22]:

$$
S_{\mathrm{NG}}=-T \int_{-\infty}^{+\infty} d \tau \int_{0}^{\pi} d \sigma \mathbf{L}=-T \int_{-\infty}^{+\infty} d \tau \int_{0}^{\pi} d \sigma \sqrt{\left(\dot{X} X^{\prime}\right)^{2}-(\dot{X})^{2}\left(X^{\prime}\right)^{2}}-T \int_{-\infty}^{+\infty} d \tau \int_{0}^{\pi} d \sigma e B_{\mu \nu} \dot{X}^{\mu} X^{\prime \nu}
$$

From the variation of this action, we obtain the following equations of motion and the boundary conditions:

$$
\begin{array}{r}
\frac{\partial P_{\mu}}{\partial \tau}+\frac{\partial \Pi_{\mu}}{\partial \sigma}=0 \\
\left.\Pi_{\mu}\right|_{\sigma=0, \pi}=0 .
\end{array}
$$

where:

$$
\begin{aligned}
P_{\mu} & =-\frac{\partial \mathbf{L}}{\partial \dot{X}_{\mu}}=-T \mathbf{L}^{-1}\left(\left(\dot{X} X^{\prime}\right) X_{\mu}^{\prime}-\left(X^{\prime}\right)^{2} \dot{X}_{\mu}\right) \\
& +T e B_{\mu \nu} X^{\prime} \nu \\
\Pi_{\mu} & =-\frac{\partial \mathbf{L}}{\partial X_{\mu}^{\prime}}=-T \mathbf{L}^{-1}\left(\left(\dot{X} X^{\prime}\right) \dot{X}_{\mu}-(\dot{X})^{2} X_{\mu}^{\prime}\right) \\
& -T e B_{\mu \nu} \dot{X}^{\nu} .
\end{aligned}
$$

Note that $P_{\mu}$ is the canonically conjugate momentum to $X_{\mu}$, which satisfies the identities:

$$
\begin{aligned}
& \left(P-T e B_{\mu \nu} X^{\prime \nu}\right)^{2}+T^{2}\left(X^{\prime}\right)^{2}=0 \\
& P_{\mu} X^{\prime \mu}=T e B_{\mu \nu} X^{\prime \mu} X^{\prime \nu}
\end{aligned}
$$


So the equation of motion is given by:

$$
\begin{aligned}
& \frac{\partial}{\partial \tau}\left[\frac{\left(\dot{X} X^{\prime}\right) X_{\mu}^{\prime}-\left(X^{\prime}\right)^{2} \dot{X}_{\mu}}{\sqrt{\left(\dot{X} X^{\prime}\right)^{2}-(\dot{X})^{2}\left(X^{\prime}\right)^{2}}}\right] \\
& +\frac{\partial}{\partial \sigma}\left[\frac{\left(\dot{X} X^{\prime}\right) \dot{X}_{\mu}-(\dot{X})^{2} X_{\mu}^{\prime}}{\sqrt{\left(\dot{X} X^{\prime}\right)^{2}-(\dot{X})^{2}\left(X^{\prime}\right)^{2}}}\right]=0 .
\end{aligned}
$$

At this point the main problem in the classical theory is that the equation of motion is highly nonlinear and incredibly complicated to solve.

What is to be done at this stage is to impose the orthonormal gauge, that is a choice of the parametrization of the string worldsheet.

The Hessian Matrix is:

$$
W^{\mu \nu}=\frac{\partial^{2} L}{\partial \dot{X}_{\mu} \partial \dot{X}_{\nu}}=\frac{T X^{\prime 2}}{(-h)^{\frac{3}{2}}}\left[-h \eta^{\mu \nu}+\left(X^{\prime}\right)^{2} \dot{X}^{\mu} \dot{X}^{\nu}+\dot{X}^{2} X^{\prime \mu} X^{\prime \nu}-\left(\dot{X} X^{\prime}\right)\left(\dot{X}^{\mu} X^{\prime \nu}+X^{\prime \mu} \dot{X}^{\nu}\right)\right]
$$

$\dot{X}^{\mu}(\tau, \sigma)$ and $X^{\prime \mu}(\tau, \sigma)$ are the null eigenvectors of the Hessian for every value of $\sigma$ except the end values $\sigma=0, \pi$. The non-null eigenvalues are degenerate for $\sigma \neq 0, \pi$ and are equal to:

$$
\frac{T\left(X^{\prime}\right)^{2}(\tau, \sigma)}{\sqrt{-h(\tau, \sigma)}} .
$$

The variational principle for the action (23), with variations $\delta_{0} X^{\mu}(\tau, \sigma)$ vanishing at $\tau=\tau_{1}, \tau_{2}$ is:

$$
\delta S=\int_{\tau 1}^{\tau 2} d \tau \int_{0}^{\pi} d \sigma L_{\mu} \delta_{0} X^{\mu}-\left.\int_{\tau 1}^{\tau 2} d \tau \Pi_{\mu} \delta_{0} X^{\mu}\right|_{0} ^{\pi}=0
$$

and gives the following Euler-Lagrange equations and boundary conditions:

$$
\begin{aligned}
& L^{\mu}(\tau, \sigma)=\dot{P}^{\mu}+\Pi^{\prime \mu} \\
& =-W^{\mu \nu}\left[\ddot{X}_{\nu}+\frac{1}{X^{\prime 2}}\left(\dot{X}^{2} X_{\nu}^{\prime \prime}-2\left(\dot{X} X^{\prime}\right) \dot{X}_{\nu}^{\prime}\right)\right] \\
& =T \partial_{\alpha}\left(\sqrt{-h} h^{\alpha \beta} \partial_{\beta} X^{\mu}\right)=0 .
\end{aligned}
$$

$\left.\Pi_{\mu}\right|_{0} ^{\pi}=T \mathbf{L}^{-1}\left((\dot{X})^{2} X_{\mu}^{\prime}-\left(\dot{X} X^{\prime}\right) \dot{X}_{\mu}\right)-\left.T e B_{\mu \nu} \dot{X}^{\nu}\right|_{0} ^{\pi}=0$

We have:

$$
\left.\Pi_{\mu} X^{\prime \mu}\right|_{0} ^{\pi}=\sqrt{-h}-\left.e B_{\mu \nu} \dot{X}^{\mu} X^{\prime \nu}\right|_{0} ^{\pi}=0
$$

The restriction from (40) to (41) is due to the requirement that a variation of the boundaries must not violate the condition $-h \geq 0$; instead, in the frequently used orthogonal gauge (O.G.) it is defined by a choice of parameters which satisfy, besides $-h(\tau, \sigma) \geq 0$, the conditions

$$
\begin{aligned}
& \dot{X}^{2}+X^{\prime 2}=\left(\dot{X} X^{\prime}\right)=0 \Rightarrow \\
& \Rightarrow h_{a b}=\dot{X}^{2}\left(\begin{array}{cc}
1 & 0 \\
0 & -1
\end{array}\right), \quad \dot{X}^{2} \geq 0 .
\end{aligned}
$$

The equation of motion now is reduced to the well-known D'Alembert equation:

$$
L^{\mu}=T\left(\ddot{X}^{\mu}-X^{\prime \prime \mu}\right)=0 \Rightarrow \ddot{X}^{\mu}-X^{\prime \prime \mu}=0,
$$

with the Boundary Conditions (BC), the Hessian matrix and the canonical conjugate momentum to $X^{\mu}$ respectively

$$
\begin{aligned}
& \left.\Pi^{\mu}\right|_{0} ^{\pi}=X^{\prime \mu}+\left.B_{\mu \nu} \dot{X}^{\nu}\right|_{0} ^{\pi}=0, \\
& W^{\mu \nu}=-\frac{T}{\dot{X}^{2}}\left(\dot{X}^{2} \eta^{\mu \nu}-\dot{X}^{\mu} \dot{X}^{\nu}+X^{\prime \mu} X^{\prime \nu}\right), \\
& \Pi^{\mu}=T\left(\dot{X}+B_{\mu \nu} X^{\prime \nu}\right) .
\end{aligned}
$$

At the end points, the usual conditions of the O.G. are

$$
X^{\prime \mu}(\tau, 0)=X^{\prime \mu}(\tau, \pi)=0 \Rightarrow \dot{X}^{2}(\tau, 0)=\dot{X}^{2}(\tau, \pi)=0 .
$$

This in particular means that at the end points the induced metric $h_{a b}$ has a zero rank. Let us remark that if one chooses coordinates such that

$$
\dot{X}^{2}(\tau, \sigma) \geq 0, \quad X^{\prime 2}(\tau, \sigma) \leq 0,
$$

then the condition $h=0$ implies two possible situations at the end points:

1. $X^{\prime 2}<0, \dot{X}^{2}=0,\left(\dot{X} X^{\prime}\right)=0$, and $\dot{X}^{\mu}$ and $X^{\prime \mu}$ independent and $\dot{X}^{\mu} \neq 0$. This is a regular case (the Jacobian of the map $(\tau, \sigma) \Rightarrow X^{\mu}$ has a maximal rank 2). In this case the rank of the induced metric $h_{a b}$ is 1 , and the end points of the string describe null surfaces [23]. There is the possible case $\dot{X}^{\mu}=0$, which is a singular case (the Jacobian of the map $(\tau, \sigma) \Rightarrow X^{\mu}$ has rank 1$)$. 
2. $\dot{X}^{\prime 2}=0, \dot{X}^{2}=0,\left(\dot{X} X^{\prime}\right)=0$, with $X^{\prime \mu}$ collinear to $\dot{X}^{\mu}$. This is a singular case (where we may have $\Pi^{\mu} \neq 0$ as well as $\left.\Pi^{\mu}=0\right)$.

The case $X^{\prime \mu}=0$, corresponding to the O.G., may be considered as a particular case of 2): O.G. is a singular case.

As a consequence, to describe the solutions of the classical equations of motion in a class of gauges including as a special case the O.G., we have to work with class 2), that is necessarily with singular coordinates.

Let us check if the boundary condition $\left.h\right|_{0} ^{\pi}=0$ is preserved in a singular case (since it was deduced in the regular one), We may perform a transformation from regular coordinates to those which will become singular at the end points, in the interior of the interval $(0, \pi)$, that is from the class 1) to the class 2). As shown in ref.[24] and more explicitly in sect. I1 of appendix I of Ref. [25], the Jacobian of the transformation vanishes as $\sqrt{\sigma}$ in $\sigma=0$ (and in an analogous way in $\pm \pi$ ), so ensuring, a fortiori, the vanishing of the new $h$ at the end points.

So we will assume the boundary conditions (44), with a choice of coordinates falling into class 2).

In order to completely define the physical hypotheses, we will assume that the total momentum $P^{\mu}$ of the string is such that $P^{2} \geq 0$, with $P^{\mu} \neq 0$. As shown in sect. I2 appendix I of Ref. [25], this ensures a unique solution at the end points of the string with $P^{\mu}$ and $\Pi^{\mu}$ finite.

The previous discussion and the boundary conditions (40) suggest the usefulness of the following extension from the interval $(0, \pi)$ to $(-\pi, \pi)$ :

$$
X^{\mu}(\tau, \sigma)=X^{\mu}(\tau,-\sigma)
$$

and to the real line with $2 \pi$ periodicity. Let us stress that, with this kind of boundary conditions, function $X^{\prime \mu}(\tau, \sigma)$, extended to the whole real axis, may be discontinuous in $\sigma=0, \pi$.

Using the Fourier expansion, the general form of the equation of motion (43) is given by:

$$
X^{\mu}(\tau, \sigma)=x_{0}^{\mu}+a_{0}^{\mu} \tau+b_{0}^{\mu} \sigma+\sum_{n \neq 0} \frac{e^{-i n \tau}}{n}\left(i a_{n}^{\mu} \cos (n \sigma)+b_{n}^{\mu} \sin (n \sigma)\right)
$$

By imposing boundary conditions (44) on (50) we get the following solution:

$$
X^{\mu}(\tau, \sigma)=x_{0}^{\mu}+\left(a_{0}^{\mu} \tau-B_{\nu}^{\mu} a_{0}^{\nu} \sigma\right)+\sum_{n \neq 0} \frac{e^{-i n \tau}}{n}\left(i a_{n}^{\mu} \cos (n \sigma)-B_{\nu}^{\mu} a_{n}^{\nu} \sigma \sin (n \sigma)\right) .
$$

\section{QUANTIZATION AND NONCOMUTATIVITY OF STRING END POINTS COORDINATES}

In this section, we quantize an open string attached to $D$-brane in the presence of the antisymmetric background $B$-field to see how the noncommutativity of space coordinates appears at the string end points.

We will use the solution (51) and the expression of the conjugate momentum given in (46) to obtain the commutation relation of the form (1).

The conjugate momentum is:

$$
\Pi^{\mu}=T\left(\dot{X}+B_{\mu \nu} X^{\nu}\right) .
$$

Using (52), the mode expansion for momentum is then:

$$
\Pi^{\mu}=T\left(a_{0}^{\nu}+\sum_{n \neq 0} a_{n}^{\nu} e^{-i n \tau} \cos (n \sigma)\right) M_{\nu}^{\mu},
$$

where $M_{i j}=\eta_{i j}-B_{i}{ }^{k} B_{k j}$. We define the Poisson bracket between functions $G$ and $K$ as:

$$
\{G, K\}=\Omega^{i j} \frac{\partial G}{\partial \xi^{i}} \frac{\partial K}{\partial \xi^{j}} .
$$

A symplectic form is defined as:

$$
\Omega=\frac{1}{2} \Omega_{i j} d \xi^{i} \wedge d \xi^{j}
$$

where $d \xi^{i} \wedge d \xi^{j}$ forms a basis for $\Omega$, and $d \xi^{i} \wedge d \xi^{j}=$ $-d \xi^{j} \wedge d \xi^{i}$. The Poisson bracket is obtained from the inverse of $\Omega$. For the usual field theory with fields $\phi_{a}$, we have

$$
\Omega=\int d^{d} \mathbf{x} d \Pi_{a}(t, \mathbf{x}) \wedge d \phi_{a}(t, \mathbf{x})
$$

We can also apply this method to our discussion. The symplectic form is given by

$$
\Omega=\int_{0}^{\pi} d \sigma d P_{\mu} \wedge d X^{\mu}
$$

We can use the given symplectic form. The modification to the Poisson bracket at the boundaries will be seen from a mode expansion. For consistency, we require that

$$
\frac{d \Omega}{d \tau}=0
$$


Using the equation of motion and the boundary conditions, we see that this is actually the case. We have explicitly

$$
\begin{aligned}
& \Omega=\frac{1}{2 \alpha^{\prime}}\left\{M_{i j} d p_{0}^{i} \wedge\left(d x_{0}^{j}+\frac{\pi}{2} B_{k}^{j} d p_{0}^{k}\right)\right. \\
& \left.+\sum_{n>0} \frac{-i}{n}\left(M_{i j} d a_{n}^{i} \wedge d a_{-n}^{j}+d a_{n}^{a} \wedge d a_{-n}^{a}\right)\right\} .
\end{aligned}
$$

Here we have used the symmetric property of $M_{i j}$, the antisymmetric property of wedge product $\wedge$ and the following formulas

$$
\begin{aligned}
& \int_{0}^{\pi} d \sigma \cos m \sigma=\pi \delta_{m, 0} \\
& \int_{0}^{\pi} d \sigma \cos n \sigma \cos m \sigma=\frac{\pi}{2}\left(\delta_{n, m}+\delta_{n,-m}\right), \\
& \int_{0}^{\pi} d \sigma \cos n \sigma \sin n \sigma=0 \\
& \int_{0}^{\pi} d \sigma \sin n \sigma \sin m \sigma=\frac{\pi}{2}\left(\delta_{n, m}-\delta_{n,-m}\right) .
\end{aligned}
$$

The Poisson bracket is obtained from the inverse of $\Omega$. We now do the canonical quantization by promoting all modes to operators, and make the Poinsson bracket become commutators $\{,\} \rightarrow[] /$,$i . The commutation rela-$ tions for the modes are therefore:

$$
\begin{aligned}
& {\left[\hat{a}_{n}^{i}, \hat{x}_{0}^{j}\right]=\left[\hat{a}_{n}^{i}, \hat{p}_{0}^{j}\right]=0,\left[\hat{a}_{m}^{i}, \hat{x}_{n}^{j}\right]=2 \alpha^{\prime} m M^{-2 i j} \delta m,-n} \\
& {\left[\hat{p}_{0}^{i}, \hat{p}_{0}^{j}\right]=0,\left[\hat{x}_{0}^{i}, \hat{p}_{0}^{j}\right]=i 2 \alpha^{\prime} M^{-1 i j}} \\
& {\left[\hat{x}_{0}^{i}, \hat{x}_{0}^{j}\right]=i 2 \pi \alpha^{\prime}\left(M^{-1} B\right)^{i j} .}
\end{aligned}
$$

Then we calculate the commutation relations for open string coordinates and momenta. We use commutation relations for the modes and note that

$$
\sum_{n \neq 0} f(n)=0, \text { for } f(n)=-f(-n),
$$

and that $\sum_{n \neq 0} \frac{(\sin n x)}{n}$ is an anti-periodic extension to $\pi-x$, over the range $[0,2 \pi]$,

$$
\sum_{n \neq 0} \frac{1}{n} \sin n x=\left\{\begin{array}{l}
0, \quad x=0,2 \pi . \\
\pi-x, \quad x \in(0,2 \pi) .
\end{array}\right.
$$

We obtain

$$
\begin{aligned}
& {\left[\hat{P}^{i}(\tau, \sigma), \hat{P}^{j}\left(\tau, \sigma^{\prime}\right)\right]=0 .} \\
& {\left[\hat{X}^{i}(\tau, \sigma), \hat{X}^{j}\left(\tau, \sigma^{\prime}\right)\right]=\left\{\begin{aligned}
2 \pi i \alpha^{\prime}\left(M^{-1} B\right)^{i j}, & \sigma=\sigma^{\prime}=0 \\
-2 \pi i \alpha^{\prime}\left(M^{-1} B\right)^{i j}, & \sigma=\sigma^{\prime}=\pi \\
0, & \text { otherwise }
\end{aligned}\right.} \\
& {\left[\hat{X}^{i}(\tau, \sigma), \hat{P}^{j}\left(\tau, \sigma^{\prime}\right)\right]=i \eta^{i j} \delta\left(\sigma, \sigma^{\prime}\right)}
\end{aligned}
$$

\section{CONCLUSION}

From the result, we see that the string coordinates become noncommutative at the string end points. This situation becomes even more difficult in the case of a totally noncommutative phase space-time; where the whole phase space becomes noncommutative, the noncommutativity affects not just the space coordinates but also the momentum coordinates to lead to the so-called totall noncommutativity. However, in a totally noncommutative phase space, neither the coordinate space representation nor the momentum space representations are allowed. So in order to study the physics in a totally noncommutative phase space, we may propose to use the generalisation of the phase space quantization. However, it is difficult to construct a quantum field theory in a totally noncommutative phase space. This is because we normally view fields as functions in either spacetime or momentum space. However, we are now forced to use phase space functions. We might need to first construct a quantum field theory in a usual phase space. Then the next step will by easy: as the fields are already functions of phase space, we may use a star-product to give the details of a totally noncommutative phase space. Alternatively, we may first try to understand some physics by studying quantum mechanics in a totally noncommutative phase space. This might provide some useful insights into constructing aquantum field theory in a totally noncommutative phase space. 
[1] A. Connes, Noncommutative Geometry, 1ed. (Academic Press, 1994).

[2] H. S. Snyder, Phys. Rev. 71, 38 (1947); https://doi. org/10.1103/PhysRev.71.38.

[3] K. Wray, An Introduction to String Theory, https://math. berkeley.edu/ kwray/papers/string_ theory.pdf (2011).

[4] M. B. Green, J. H. Schwarz, E. Witten, Superstring Theory, (Cambridge University Press, 1987).

[5] B. Zwiebach, A First Course in String Theory, 2nd ed. (Cambridge University Press, 2009).

[6] R. Blumenhagen, D. Lust, S. Theisen, Basic Concepts of String Theory (Theoretical and Mathematical Physics), (Springer, 2012); https://doi.org/10.1007/ 978-3-642-29497-6.

[7] E. Witten, Nucl. Phys. B 268, 253 (1986); https://doi. org/10.1016/0550-3213(86) 90155-0.

[8] R. Blumenhagen, Fortschr. Phys. 62, 709 (2014); https : //doi.org/10.1002/prop. 201400014.

[9] N. Seiberg, E. Witten, J. High Energy Phys. 1999(JHEP09), 032 (1999); https://doi.org/10.1088/ 1126-6708/1999/09/032.

[10] L. Dolan, C. R. Nappi, International Conference on Superstring Theory. 12-15 Aug 2002, Hangzhou, China. Proceedings, edited by K. Liu, S. T. Yau, C. Zhu (Somerville, International Press, 2008), p. 69; preprint arXiv:hep-th/0302122 (2003).

[11] A. Connes, M. R. Douglas, J. High Energy Phys. 1998(JHEP02), 003 (1998); https://doi.org/10.1088/ 1126-6708/1998/02/003

[12] Chong-Sun Chu, in Encyclopedia of Mathematical Physics (Academic Press, 2006), p. 515.

[13] C. Hofman, E. Verlinde, J. High Energy Phys. 1998(JHEP12), 010 (1998); https://doi.org/10.1088/
1126-6708/1998/12/010.

[14] C.-S. Chu, P.-M. Ho, Nucl. Phys. B 550, 151 (1999); https://doi.org/10.1016/S0550-3213(99)00199-6.

[15] F. Ardalan, H. Arfaei, M. M. Sheikh-Jabbari, Nucl. Phys. B 576, 578 (2000); https://doi.org/10.1016/ S0550-3213(00) 00096-1.

[16] Zheng-Wen Long, Jian Jing, Phys. Lett. B 560, 128 (2003); https://doi.org/10.1016/S0370-2693(03) $00375-7$.

[17] Won Tae Kim, J. J. Oh, Mod. Phys. Lett. A 15, 1597 (2000); https://doi.org/10.1142/S0217732300002127.

[18] Xiao-Jun, preprint arXiv:hep-th/0503111 (2005).

[19] Chong-Sun Chu, Pei-Ming Ho, Nucl. Phys. B 568, 447 (2000); https://doi.org/10.1016/S0550-3213(99) 00685-9.

[20] F. Ardalan, H. Arfaei, M. M. Sheikh-Jabbari, J. High Energy Phys. 1999(JHEP02), 016 (1999); https : //doi . org/10.1088/1126-6708/1999/02/016.

[21] S. Gangopadhyay, A. G. Hazra, A. Sahab, Phys. Rev. D 74, 125023 (2006); https://doi.org/10.1103/ PHYSREVD.74.125023.

[22] R. Banerjee, Phys. Rev. D 70, 026006 (2004); https: //doi.org/10.1103/PhysRevD.70.026006

[23] D. C. Salisbury, K. Sundermeyer, Nucl. Phys. B 191, 250 (1981); https://doi.org/10.1016/0550-3213(81) 90300-X.

[24] D. G. Boulware, E. T. Newman, Phys. Lett. B 174, 378 (1986); https://doi.org/10.1016/0370-2693(86) 91020-8.

[25] F. Colomo, G. Longhi, L. Lusanna, Int. J. Mod. Phys. A 5, 3347 (1990); https://doi.org/10.1142/ S0217751X90001471.

[26] N. Mansour, E. Y. Diaf, M. B. Sedra, Electron. J. Theor. Phys. 14, 21 (2018).

\title{
ТЕОРІЯ СТРУН У ФОРМАЛІЗМІ НАМБУ-ГОТО ТА НЕКОМУТАТИВНІСТЬ
}

\author{
Н. Мансур ${ }^{1}$, Е. Діаф ${ }^{2}$, М. Б. Седра ${ }^{1}$ \\ ${ }^{1}$ SIMO-Lab, факультет природничих наук, Університет Ібн Тофайла, Кенітра, Марокко, \\ ${ }^{2}$ Кафедра фізики, Університет Мохаммеда Першого, Надор, Марокко, \\ e-mail:nmansour@live.fr,eldiaf@gmail.com mysedra@yahoo.fr
}

У цій роботі ми вивчали теорію струн у первісній формі Набму-Гото. Встановлено, що розрахунок варіації дії Намбу-Гото за метрикою світового листа призводить до нелінійної дії, яка не є строго еквівалентною дії Полякова. Тоді рівняння руху, що відповідає цій дії, є нелінійним. Саме тому ми вирішили розв'язати нелінійне рівняння руху Намбу-Гото безпосередньо. Це було зроблено для струни зі сталим антисиметричним тензорним полем $B$ у формалізмі Намбу-Гото. Ми вивели некоммутативність між координатами кінців струни. 